\title{
Miranda
}

Revue pluridisciplinaire du monde anglophone /

Multidisciplinary peer-reviewed journal on the English-

speaking world

$12 \mid 2016$

Mapping gender. Old images ; new figures

\section{Damian Grant, Salman Rushdie romancier}

\section{Marc Porée}

\section{OpenEdition}

\section{Journals}

Édition électronique

URL : http://journals.openedition.org/miranda/7873

DOI : $10.4000 /$ miranda.7873

ISSN : 2108-6559

Éditeur

Université Toulouse - Jean Jaurès

Référence électronique

Marc Porée, «Damian Grant, Salman Rushdie romancier », Miranda [En ligne], 12 | 2016, mis en ligne le 26 février 2016, consulté le 16 février 2021. URL : http://journals.openedition.org/miranda/7873 ; DOI : https://doi.org/10.4000/miranda.7873

Ce document a été généré automatiquement le 16 février 2021.

\section{c) $8 \odot$}

Miranda is licensed under a Creative Commons Attribution-NonCommercial-NoDerivatives 4.0 International License. 


\title{
Damian Grant, Salman Rushdie romancier
}

\author{
Marc Porée
}

\section{RÉFÉRENCE}

Damian Grant, Salman Rushdie romancier, traduit de l'anglais par Madeleine Descargues (Lille : Presses Universitaires du Septentrion, 2014), 221 p, ISBN 978-2-7574-0793-6

1 Lire Salman Rushdie romancier est une expérience aussi rare qu'enrichissante, et qui se recommande grandement. Commençons par la rareté. Personne ne soutiendra qu'il n'existait pas d'étude critique digne de ce nom avant la sortie, en version française, de cet ouvrage initialement paru en 1999 chez Manchester Press. C'est même tout le contraire, tant et si bien qu'on croyait avoir tout lu et tout dit sur Rushdie, son œuvre, sa vie, sur la fatwa et ses suites, lointaines (déjà 16 ans !) et encore si proches. Mais il faut se rendre à l'évidence, Damian Grant fait œuvre puissamment inédite, en abordant la production rushdienne par des chemins rarement empruntés de nos jours. Il est rarissime, en effet, qu'on l'analyse par le biais de Shelley, et de sa vibrante Défense de la poésie, ou bien encore à la faveur du recours, particulièrement convaincant, à Jonathan Swift et à son Conte du Tonneau, à Beckett ou, cerise sur le gâteau, à W.B. Yeats (et son peu connu «High Talk»). Sans parler des références à peine moins inattendues : Sidney, Sterne, Blake, Coleridge, Keats, Dickens, Kipling, James, Joyce, Lawrence, Wallace Stevens, Ted Hughes, Pynchon, etc. La manie de la référence (à la littérature occidentale, ajoutera-t-on, quitte à noircir un peu plus l'image de Rushdie auprès de ses compatriotes restés au pays) n'est pas seule en cause.

2 C'est plutôt du côté de l'imagination créatrice qu'il faut chercher. Elle sert de fil rouge à l'ouvrage, donnant tout loisir à Grant de passer au banc d'essai les différentes œuvres de Rushdie, dans l'ordre de leur parution. L'imagination tient lieu de pierre de touche ; cette faculté qui nous permet de construire nos images du monde serait ainsi seule à même de révéler notre humanité, seule aussi à pouvoir distinguer le bon grain de 
l'ivraie, en l'occurrence, le roman pleinement réussi du récit bâclé, parce que superficiel ou répétitif. D'un mot, l'imagination sert à Grant à établir une ligne de partage des eaux au sein de l'abondante œuvre rushdienne, entre d'un côté les romans dans lesquels l'imagination rejoint l'énergie dont font preuve les protagonistes et que déploie le romancier dans leur quête commune de liberté, mais aussi de vérité et de créativité, et de l'autre, ceux qui, postérieurs à Le Dernier soupir du Maure (1995), « en même temps qu'ils cultivent le spectaculaire et le superficiel de manière générale, traitent l'imagination comme un jouet plus que comme une qualité morale; on joue avec elle et on la range dans sa boîte, mais elle a perdu la considération sérieuse que lui donnait un Shelley » (195). Ce serait le cas de La Terre sous ses pieds, de Shalimar le clown, de Luka et le Feu de la vie (dénué de «feu » et de « vie ») et, à un degré moindre, de Furie.

3 L'expérience est enrichissante ensuite, car Damian Grant, consciemment ou inconsciemment, écrit pour transmettre au lecteur son amour de la littérature et partager avec lui/elle son immense culture. Lire à sa suite décuple le plaisir pris à décrypter, à redécouvrir, à rapprocher. Ce plaisir est toujours éclairé et éclairant, tressé à part égale de savoir et de sensibilité au fait littéraire, nourri par de longues années passées à enseigner, à diriger le séminaire de littérature britannique contemporaine pour le compte du British Council, à écrire et à publier de la poésie. Sa méthode est-elle anglaise? Oui, assurément, en ce qu'elle repose sur un examen attentif et minutieux des faits, à la recherche de preuves - lesquelles peuvent être, Grant ne craindrait pas de l'admettre, des preuves d'amour, de la part d'un « adorateur de longue date » de Rushdie, mais aussi de désamour, le désenchantement s'installant de manière durable. Mais on aura reconnu quelque chose, aussi, de cette moral earnestness que cherchaient un Matthew Arnold (avec son «criticism of life») et plus tard un F.R. Leavis (adepte de la grande tradition du roman anglais). A ceci près que Grant est décidément trop frotté de romantisme et d'idéalité (Shelley, Coleridge, Keats, Blake, Wordsworth) pour n'être que froidement leavisite.

4 Ajoutons qu'il sait également mettre de la francité dans son vin anglais. Il convient en effet de revenir sur le titre que s'est choisi Grant: Salman Rushdie romancier. La chose paraît aller de soi, mais il n'en est rien. Et Grant a raison de relever que la critique rushdienne, pour prolifique qu'elle soit, se cantonne essentiellement à un registre idéologique, théorique ou politique (Timothy Brennan, Aijaz Ahmad, Andrew Teverson). Or, c'est à l'entreprise fictionnelle d'édification d'une existence en concurrence avec la réalité « vraie », que s'intéresse prioritairement Grant, spécialiste $\mathrm{du}$ roman du XVIII ${ }^{\mathrm{e}}$ siècle et auteur, dans sa jeunesse, d'une brève et pertinente synthèse sur le Réalisme (parue chez Methuen en 1970). C'est avec l'écriture qu'il argumente, pied à pied, dans le sillage, mais le sait-il ?, de l'ouvrage de Catherine PessoMiquel, Salman Rushdie : l'écriture transportée (Presses Universitaires de Bordeaux, 2007). C'est avec elle - l'écriture, toujours - qu'il traite, et s'il se montre intraitable, voire implacable, c'est en raison même du degré d'engagement et d'attention soutenue que Grant accorde à l'immense auteur des Enfants de Minuit et des Versets Sataniques.

5 La deuxième partie de l'ouvrage, rédigée pour la nouvelle édition anglaise de 2012, voit le critique s'agacer, et ne pas parvenir, in fine, à modérer l'expression de sa forte désapprobation. Les analyses tournent à l'exécution en règle, le lecteur se fait procureur. Un instant, devant la sévérité parfois extrême du jugement, on se sent porté à prendre la défense de l'écrivain, mais la rigueur avec laquelle Grant s'acquitte de sa tâche ne laisse planer aucun doute, s'agissant en tout cas de la parfaite objectivité du 
diagnostic. En maintenant de bout en bout le même protocole méthodologique, consistant à reprendre quasi exhaustivement la trame de chacun des romans étudiés, Grant fait ressortir dans une lumière encore plus crue la «transformation radicale de l'écriture » (163), impuissante à endiguer la montée en puissance d'un certain nombre de travers difficilement supportables: manque de discernement et de réserve, complaisance et narcissisme, hystérisation du rapport à la réalité, verbiage. L'Enchanteresse de Florence est le seul roman récent à trouver grâce aux yeux de Grant, qui y retrouve la joie et l'innocence du conteur, au motif que ce dernier se trouve protégé «du bruit assourdissant, du carnaval des noms inventés ou réels, des inventaires journalistiques et des égarements visuels du vingt et unième siècle » (196).

6 Faut-il alors craindre le pire en songeant que c'est précisément dans le cadre d'un New York à peine futuriste que Rushdie situe l'intrigue de Two Years, Eight Months and Twenty-Eight Nights, son dernier roman en date? Rushdie saura-t-il se garder de ses mauvais démons? On le souhaite ardemment, au nom des éclatantes réussites passées. La conclusion de son ouvrage voit Damian Grant s'adresser, bien sûr au lecteur, mais aussi à Salman Rushdie le romancier, dans l'espoir que ce dernier l'entendra lui rappeler que «c'est sur son œuvre la plus forte, celle qui survit au temps et qui laisse son empreinte dans l'imagination, qu'il sera en fin de compte jugé de la façon la plus équitable» (201). A bon entendeur, salut...

\section{INDEX}

Keywords : Salman Rushdie, the novel, the creative imagination, fiction

Mots-clés : Salman Rushdie, roman, imagination créatrice, fiction

\section{AUTEURS}

\section{MARC PORÉE}

Professeur des Universités

ENS

marc.poree@ens.fr 